\title{
Effects of the quark-hadron phase transition on highly magnetized neutron stars
}

\author{
B. Franzon, ${ }^{1 \star}$ R. O. Gomes ${ }^{2 \star}$ and S. Schramm ${ }^{3 \star}$ \\ ${ }^{1}$ Frankfurt Institute for Advanced Studies, Ruth-Moufang - 1, D-60438, Frankfurt am Main, Germany \\ ${ }^{2}$ Instituto de Física, Universidade Federal do Rio Grande do Sul, Porto Alegre-RS 91501-970, Brazil \\ ${ }^{3}$ Frankfurt Institute for Advanced Studies, Ruth-Moufang - 1, D-60438, Frankfurt am Main, Germany
}

Accepted 2016 August 4. Received 2016 July 2; in original form 2016 April 26

\begin{abstract}
The presence of quark-hadron phase transitions in neutron stars can be related to several interesting phenomena. In particular, previous calculations have shown that fast rotating neutron stars, when subjected to a quark-hadron phase transition in their interiors, could give rise to the backbending phenomenon characterized by a spin-up era. In this work, we use an equation of state composed of two phases, containing nucleons (and leptons) and quarks. The hadronic phase is described in a relativistic mean field formalism that takes many-body forces into account, and the quark phase is described by the MIT bag model with a vector interaction. Stationary and axisymmetric stellar models are obtained in a self-consistent way by solving numerically the Einstein-Maxwell equations by means of a pseudo-spectral method. As a result, we obtain the interesting backbending phenomenon for fast spinning neutron stars. More importantly, we show that a magnetic field, which is assumed to be axisymmetric and poloidal, can also be enhanced due to the phase transition from normal hadronic matter to quark matter on highly magnetized neutron stars. Therefore, in parallel to the spin-up era, classes of neutron stars endowed with strong magnetic fields may go through a 'magnetic-up era' in their lives.
\end{abstract}

Key words: equation of state-gravitational waves - magnetic fields.

\section{INTRODUCTION}

Compact stars refer collectively to white dwarfs (WD), neutron stars (NS), or even black holes. The latter two are usually formed in catastrophic astrophysical events such as supernova explosions, see e.g. Glendenning (2012) and Shapiro \& Teukolsky (2008). In a few seconds, these explosions release the brightness of millions of suns, so it is not surprising that such phenomena have been observed since ancient times. The kind of the remnant depends primarily on the mass of the proto-star. Moreover, these objects harbour compressed ultradense matter in their interiors, have spin rotation from zero to milliseconds and can support ultrahigh magnetic fields. These features, in conjunction with the corresponding progress in observational astrophysics, make them one of the most and, sometimes, the only suitable environment to inspect, or at least to infer from, the behaviour of matter and electromagnetic fields under extreme conditions.

Depending on the equation of state (EoS) chosen to describe the matter, the density inside NS can reach values several times higher than the nuclear saturation density $\rho_{0} \sim 0.15 \mathrm{fm}^{-3}$. In fact, during

${ }^{\star}$ E-mail: brfranzon@gmail.com (BF); rosana.gomes@ufrgs.br (ROG); schramm@fias.uni-frankfurt.de (SS) the past years, much effort has been made in order to describe and shed light on the still open question concerning the EoS for ultradense nuclear matter. Furthermore, another important question that we address in this work is the impact of the magnetic field on the structure and on the evolution of these stars. The knowledge of the microscopic theory related to the EoS allows not only to study the general structure of the star, including its deformation due to the magnetic field and due to rotation, but also provides information about the inner composition of the star, including possible exotic phases such as quarks and hyperon in the stellar core.

As shown in Glendenning, Pei \& Weber (1997) and Zdunik et al. (2006), phase transitions inside NS can be accessed through the backbending phenomenon, where stars spin-up over time as a consequence of a phase change in their cores. However, the exact mechanism responsible for the spin-down of stars is still unclear. The most accepted idea is that these stars spin-down because of magnetic torques and lose energy through magnetic dipole radiation (MDR). In this way, rotating magnetized stars behave as oblique rotators (Pacini 1967, 1968; Gold 1975). In addition to the dipole radiation, processes such as emission of gravitational radiation and pulsar wind can also contribute to the braking index of NS (Ostriker \& Gunn (1969), Ferrari \& Ruffini (1969), Blandford \& Romani (1988), Manchester, Durdin \& Newton (1985)). In all cases, the star energy loss can be described by a power law $\dot{E}=-C \Omega^{n+1}$, 
$C$ being a term that accounts for the pulsar structure, $E$ for the kinetic energy, $\Omega$ is the pulsar angular velocity and $n$ is the braking index, which therefore describes the dependence of the braking torque on the rotation frequency.

According to the MDR theory, a rigid star with a constant dipole magnetic field and a constant moment of inertia $I$ has the canonical braking index of $n=3$. As calculated in Pacini $(1967,1968)$, the spin-down relation for such stars is given by $\dot{\Omega} \propto \Omega^{3}$. However, in the presence of rotation, the moment of inertia $I$ is not constant in time and, therefore, one has to considerer the dependence $I(\Omega)$. In this case, the star responds to changes due to the centrifugal force becoming oblate. This effect reduces the value of the braking index from the standard oblique rotator model to a value of $n<3$, see Hamil et al. (2015). Furthermore, the microphysics impacts strongly the braking index, which can have values in the range of $-\infty<n<\infty$ in presence of a first-order phase transition, see e.g. Glendenning et al. (1997) and Chubarian et al. (2000).

In case of a phase transition, its duration epoch is governed by a slower loss of the star angular momentum due to radiation. This will be seen in the behaviour of the $I(\Omega)$ curve presented in Section 3. Similar results as those presented in Section 3 were already investigated before, see for example Glendenning et al. (1997), Zdunik et al. (2006) and Chubarian et al. (2000). At the same time, as we will see in Section 4, this phase change in the stellar interior can introduce not only a spin-up era, but also an increase in the magnetic field throughout the star.

From pulsar spin-down observations together with the magnetic dipole model, the magnitude of the surface magnetic field in NS is typically estimated to be of the order of $10^{12}-10^{13} \mathrm{G}$. However, other classes of NS known as anomalous X-ray pulsars and soft gammaray repeater, referred to magnetars, can have surface magnetic fields as large as $10^{14}-10^{15} \mathrm{G}$ (Duncan \& Thompson 1992; Paczynski 1992; Thompson \& Duncan 1993, 1996; Melatos 1999). Such strong magnetic fields affect both the structure and the composition of these stars and can, potentially, convert a hybrid star into a hadronic star as shown in Franzon, Dexheimer \& Schramm (2015).

By modelling rapidly rotating NS, we show the effects of a phase transition on the moment of inertia $I(\Omega)$ of the stars and, as a consequence, the impact on the braking index $n(\Omega)$ (which becomes frequency-dependent). In addition, we constructed models for highly magnetized NS and we show that, similarly to the rotating case, the magnetic field induces a reduction in the moment of inertia, which introduces an era in the stellar evolution where the magnetic field increases. In comparison with the spin-up era for fast rotating bodies, we call this phenomenon a 'magnetic-up era'.

\section{EQUATION OF STATE}

The transition from confined to deconfined matter inside NS has been extensively studied over the last years and, in many cases, also applied to study properties of hybrid stars (Bombaci et al. 2007, 2009; Yasutake et al. 2011; Lenzi \& Lugones 2012; Ayvazyan et al. 2013; Brillante \& Mishustin 2014; Alvarez-Castillo \& Blaschke 2015; de Carvalho et al. 2015; Dexheimer et al. 2015; Franzon et al. 2015). Despite this progress, at the present moment, there are still substantial uncertainties in the EoS regarding the description of the stellar matter at supra-nuclear densities.

In this work, we use an EoS with quark-hadron phase transition to describe the stellar interior. The hadronic phase, composed of nucleons (together with leptons), is described in the framework of a relativistic mean-field theory and takes into account many-body forces contributions in the baryon couplings. For the quark phase,
Table 1. Properties of the particles used in the formalism: mass $m$, projection of the isospin in the $z$ direction $I^{3}$, baryon charge $q_{\mathrm{b}}$ and electric charge $q_{\mathrm{e}}$.

\begin{tabular}{lcccc}
\hline Particle & Mass $(\mathrm{MeV})$ & $I^{3}$ & $q_{\mathrm{b}}$ & $q_{\mathrm{e}}$ \\
\hline$p$ & 939.6 & $1 / 2$ & 1 & +1 \\
$n$ & 938.3 & $-1 / 2$ & 1 & 0 \\
$e^{-}$ & 0.511 & 0 & 0 & -1 \\
$\mu^{-}$ & 105.7 & 0 & 0 & -1 \\
$u$ & 1.0 & - & $1 / 3$ & $+2 / 3$ \\
$d$ & 1.0 & - & $1 / 3$ & $-1 / 3$ \\
$s$ & 100.0 & - & $1 / 3$ & $-1 / 3$ \\
\hline
\end{tabular}

Table 2. Meson fields properties considered in the hadronic phase and their respective coupling constants at nuclear saturation density.

\begin{tabular}{llcr}
\hline Meson & Classification & $\begin{array}{c}\text { Mass } \\
(\mathrm{MeV})\end{array}$ & \begin{tabular}{c}
$\left(g_{\left.i_{N} / m_{i}\right)^{2}}\left(\mathrm{fm}^{2}\right)\right.$ \\
\hline$\sigma$
\end{tabular} \\
$\delta$ & scalar-isoscalar & 550 & 14.51 \\
$\omega_{\mu}$ & scalar-isovector & 980 & 0.38 \\
$\varrho$ & vector-isoscalar & 782 & 8.74 \\
\hline
\end{tabular}

we use the MIT bag model with vector interaction, in order to reproduce the maximum effect that phase transitions can produce. We choose in this work to describe the deconfinement phase transition by using the usual Maxwell construction, i.e. reproduce a sharp phase transition.

\subsection{Hadronic phase}

The Lagrangian density of the MBF model reads:

$$
\begin{aligned}
\mathcal{L}= & \sum_{\mathrm{b}} \bar{\psi}_{\mathrm{b}}\left[\gamma_{\mu}\left(i \partial^{\mu}-g_{\omega} \mathrm{b} \omega^{\mu}-g_{\varrho \mathrm{b}} \mathrm{I}_{3 \mathrm{~b}} \varrho_{3}^{\mu}\right)-m_{\mathrm{b} \zeta}^{*}\right] \psi_{\mathrm{b}} \\
& +\left(\frac{1}{2} \partial_{\mu} \sigma \partial^{\mu} \sigma-m_{\sigma}^{2} \sigma^{2}\right)+\frac{1}{2}\left(-\frac{1}{2} \omega_{\mu \nu} \omega^{\mu \nu}+m_{\omega}^{2} \omega_{\mu} \omega^{\mu}\right) \\
& +\frac{1}{2}\left(-\frac{1}{2} \varrho_{\mu \nu} \cdot \varrho^{\mu \nu}+m_{\varrho}^{2} \varrho_{\mu} \cdot \varrho^{\mu}\right)+\left(\frac{1}{2} \partial_{\mu} \delta . \partial^{\mu} \boldsymbol{\delta}-m_{\delta}^{2} \boldsymbol{\delta}^{2}\right) \\
& +\sum_{1} \bar{\psi}_{1} \gamma_{\mu}\left(i \partial^{\mu}-m_{1}\right) \psi_{1} .
\end{aligned}
$$

The subscripts $b$ and $l$ correspond to nucleonic $(n, p)$ and lepton $\left(e^{-}, \mu^{-}\right)$degrees of freedom. The first and last terms in equation (1) represent the Dirac Lagrangian density for nucleons and leptons. The other terms represent the Lagrangian densities of the scalar mesons $\sigma$ and $\delta$ (Klein-Gordon Lagrangian density), and the vector mesons $\omega$ and $\varrho$ (Proca Lagrangian density). The meson-baryon interaction appears in the first term, contained in the coupling constants $\left(g_{\omega \mathrm{b}}, g_{\varrho \mathrm{b}}\right)$ and effective masses $\left(m_{\mathrm{b} \zeta}^{*}\right)$. The properties of the particles used in this work can be found in the Tables 1 and 2. In this formalism, the $\delta$ and $\varrho$ fields allow for a better description of the isospin asymmetry of the system. See Alaverdyan, Alaverdyan \& Chiladze (2010) for an analysis of the influence of the delta meson on phase transitions.

The effects of the many-body forces contribution (controlled by the $\zeta$ parameter) and the nuclear interaction on the baryonic effective masses and chemical potentials are expressed in the following

$m_{\mathrm{b}}^{*}=m_{\mathrm{b}}-\left(1+\frac{g_{\sigma \mathrm{b}} \sigma+g_{\delta \mathrm{b}} I_{3 \mathrm{~b}} \delta_{3}}{\zeta m_{\mathrm{b}}}\right)^{-\zeta}\left(g_{\sigma \mathrm{b}} \sigma+g_{\delta \mathrm{b}} I_{3 \mathrm{~b}} \delta_{3}\right)$ 
$\mu_{\mathrm{b}_{i}}^{*}=\sqrt{k_{f_{\mathrm{b}}}^{2}+\left(m_{\mathrm{b} \zeta}^{*}\right)^{2}}+g_{\omega \mathrm{b}} \omega+g_{\varrho \mathrm{b}} I_{3 \mathrm{~b}} \varrho_{3}+g_{\phi \mathrm{b}} \phi$,

where $k_{f_{\mathrm{b}}}$ and $m_{\mathrm{b}}$ correspond to the fermi momenta and the bare masses of the baryons.

By choosing small values of the $\zeta$ parameter, the second term in equation (2) can be expanded by means of nonlinear self-couplings of the scalar fields $(\sigma, \delta)$, simulating the effects of many-body forces in the nuclear interaction. Each set of parameters generates different EoS's and, hence, different sets of nuclear saturation properties. For this study, we use the parametrization: $\zeta=0.040$, reproducing a binding energy of $B / A=-15.75 \mathrm{MeV}$ and the saturation density $\rho_{0}=0.149 \mathrm{fm}^{-3}$. This set of parameters reproduces the following nuclear saturation properties: effective mass of the nucleon $m^{*} / m=0.66$, incompressibility $K_{0}=297 \mathrm{MeV}$, symmetry energy $J_{0}=32 \mathrm{MeV}$ and slope of the symmetry energy $L_{0}=97 \mathrm{MeV}$ (for more details of nuclear saturation properties covered by the MBF model, see e.g. Gomes et al. 2015).

\subsection{Quark phase}

For the quark phase, we adopt the MIT bag model with an additional vector interaction. We take the quarks $u, d$ and $s$, and the leptons $e^{-}$and $\mu^{-}$as degrees of freedom (see Table 1). The Lagrangian density of the model reads:

$$
\begin{aligned}
\mathcal{L}= & \sum_{\mathrm{q}}\left[\bar{\psi}_{\mathrm{q}}\left(i \gamma_{\mu} \partial^{\mu}-g_{V \mathrm{q}} \gamma_{\mu} V^{\mu}-m_{\mathrm{q}}-B\right) \psi_{\mathrm{q}}\right] \theta_{\mathrm{H}} \\
& +\sum_{1} \bar{\psi}_{1} \gamma_{\mu}\left(i \partial^{\mu}-m_{1}\right) \psi_{\mathrm{l}},
\end{aligned}
$$

with $\theta_{\mathrm{H}}$ being the Heavyside function responsible for the confinement feature of the model $\left(\theta_{\mathrm{H}}=1\right.$ inside the bag; $\theta_{\mathrm{H}}=0$ outside), and $B$ is the bag constant. This constant represents the extra energy per unit of volume required to create a region of perturbative vacuum (Farhi \& Jaffe 1984).

The vector interaction is introduced by a vector-isoscalar meson $V^{\mu}$, with coupling constant $g_{\mathrm{V}}$, coupling to all three quarks. As discussed in Shao et al. (2013), the field that introduces the vector interaction can be considered analogous to the $\omega$ field used in the hadronic model. As in the hadronic phase, the vector interaction introduces a shift in the quark chemical potential:

$\mu_{\mathrm{q}_{i}}^{*}=\sqrt{k_{f_{\mathrm{q}}}^{2}+m_{\mathrm{q}}^{2}}+g_{\mathrm{Vq}} V$.

The vector interaction has been extensively studied in the context of bag models (Contrera et al. 2014; Klähn \& Fischer 2015) and Nambu-Jona-Lasinio models (Contrera et al. 2014; Klähn \& Fischer 2015; Ranea-Sandoval et al. 2016), and has been applied to different investigations such as the study of the phase structure and transitions of matter (Shao et al. 2012; Contrera et al. 2014), the effect of strong magnetic fields (Denke \& Pinto 2013; Menezes et al. 2014), the thermal evolution of NS (de Carvalho et al. 2015) and others. In particular, the approach used in this work is analogous to the one proposed in Alford et al. (2005) and Weissenborn et al. (2011), which introduces phenomenological corrections based on gluon effects that result in extra terms in the EoS and particles densities of quark matter. Note that the introduction of a vectorisovector field generates these same shifts in the EoS, due to the additional term, $V^{\mu}$, in the pressure, as well as in the energy density. In addition, $V^{\mu}$ changes the particle population, as the quark chemical potentials are modified.

In this context, the hardest task of such models is to determine the coupling constant of the vector interaction. There are attempts

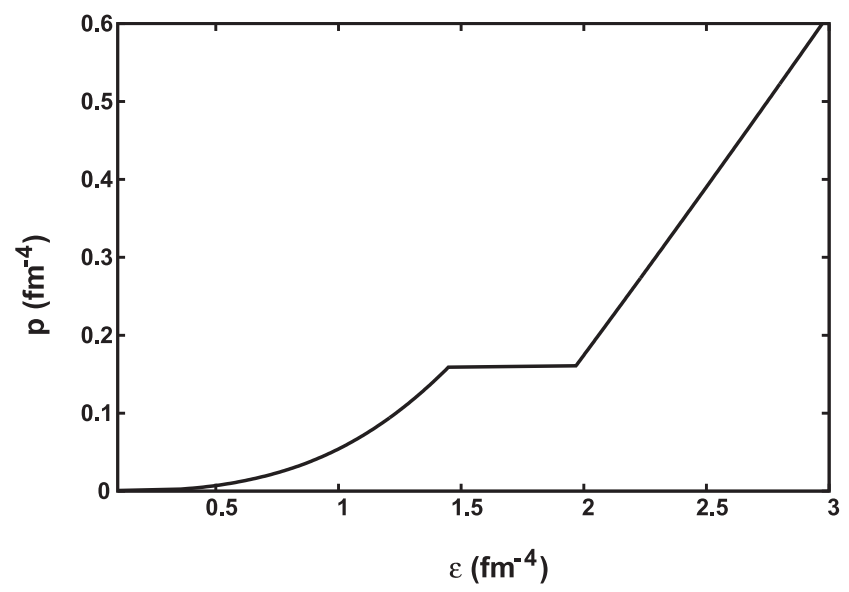

Figure 1. EOS for neutron star matter including a deconfinement phase transition for the parametrization $\zeta=0.040$ of the MBF formalism. The bag constant is $B=160 \mathrm{MeV}^{4}$ and the vector coupling $\left(g_{\mathrm{V}} / m_{\mathrm{V}}\right)^{2}=2.2 \mathrm{fm}^{2}$.

to account for the interactions of quarks and gluons and, then, constrain the value of the coupling by incorporating higher orders of perturbation theory and radiative corrections as done in Fraga, Pisarski \& Schaffner-Bielich (2001), Fraga, Kurkela \& Vuorinen (2014) and Restrepo et al. (2015). However, such couplings remain widely uncertain and may also have a dependence on the density and temperature.

To conclude, we take the values of the vector coupling and bag constant to be: $\left(g_{\mathrm{V}} / m_{\mathrm{V}}\right)^{2}=2.2 \mathrm{fm}^{2}, B^{1 / 4}=160 \mathrm{MeV}$, in order to reproduce massive and stable hybrid stars. The choice of parameters of both phases permits the complete EoS to describe the symmetric nuclear matter in terms of a pure hadronic phase at low densities, and the regime of high density reached in the inner core of NS to be described by a quark phase.

\subsection{Phase transition}

In this section, we describe the hadron-quark phase transition in the interior of hybrid stars by using a Maxwell construction. In this case, both phases are charge neutral and the conditions on the chemical equilibrium determine the co-existence phase. This transition is described by a regime of constant pressure, which leads to a discontinuity in the energy density and in the baryon number density. The Maxwell criteria read:

$P_{\mathrm{H}}=P_{\mathrm{Q}}, \quad \mu_{n}^{\mathrm{H}}=\mu_{n}^{\mathrm{Q}}$.

For the parametrizations used in this work, we found that the phase transition occurs at $\mu_{n}=1101.3 \mathrm{MeV}$, which corresponds to a transition pressure of $P_{0}=0.16 \mathrm{fm}^{-4}$ and an energy gap of $\Delta \varepsilon=0.52 \mathrm{fm}^{-4}$. The EoS including both phases is depicted in Fig. 1 .

The issue of whether the phase transition takes place in a Maxwell or a Gibbs scenario depends on the surface tension between the two phases. The comparison of both scenarios in the investigation of hybrid stars has been studied in several works, see e.g. Bhattacharyya, Mishustin \& Greiner (2010), Hempel, Pagliara \& Schaffner-Bielich (2009), Yasutake \& Kashiwa (2009), Yasutake et al. (2011) and Alaverdyan et al. (2010). Furthermore, the threshold values of the surface tension necessary to describe each type of transition scenario have been calculated in Lugones, Grunfeld \& Al Ajmi (2013) and Garcia \& Pinto (2013). However, such estimations are highly 


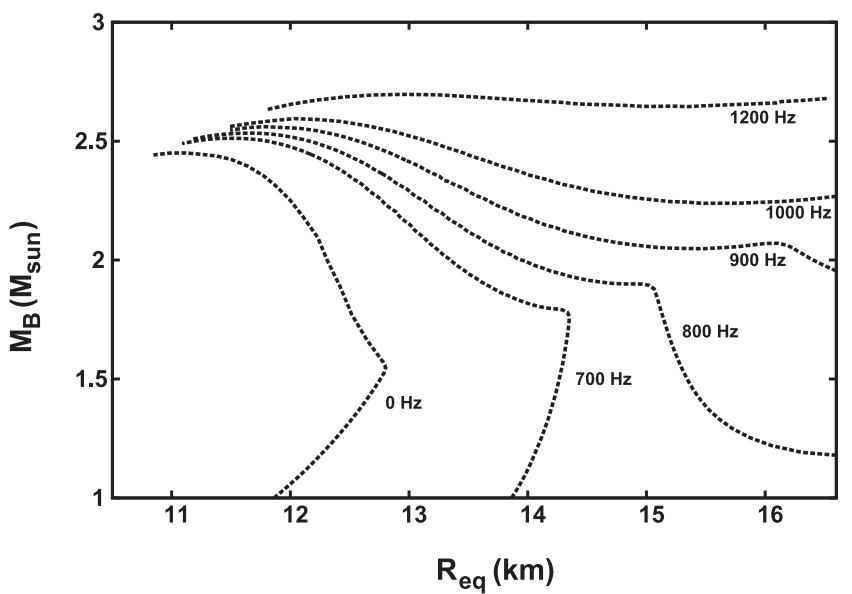

Figure 2. Baryon mass versus equatorial radius for neutron stars at different fixed rotation frequencies. These configurations were calculated using the EoS presented in Fig. 1.

model-dependent, and the issue of possible phase transition scenarios remains an open question.

\section{ROTATING HYBRID STARS}

Rapidly rotating general relativistic stars were already described in Komatsu, Eriguchi \& Hachisu (1989), Cook, Shapiro \& Teukolsky (1992) and Stergioulas \& Friedman (1995). In this section, we calculate stationary equilibrium configurations of uniformly rotating cold NS within a general relativity framework. We solve the Einstein field equations for stationary axisymmetric space-time using the $\mathrm{C}++$ class library for numerical relativity, LORENE (http://www.lorene.obspm.fr). The formalism, the relevant equations, the numerical procedure and tests used to construct the stellar models can be found in Gourgoulhon (2012), Bonazzola et al. (1993), Bocquet et al. (1995) and Chatterjee et al. (2015). Recently, this formalism was applied to magnetized hybrid stars and magnetized and fast rotating WD, see e.g. Franzon et al. (2015) and Franzon \& Schramm (2015).

The internal composition of rotating NS is modelled by the EoS as described in Section 2. The dependence of the internal structure of the NS with rotation is crucial, since the centrifugal force due to the rotation will help to stabilize the star against collapse and the star will be deformed: compressed in the polar direction and expands in the equatorial direction. With this in mind, different rotation frequencies produce different relations between the mass and the radius for rapidly rotating stars as shown in Friedman \& Ipser (1992), Spyrou \& Stergioulas (2002), Zdunik et al. (2008), Haensel et al. (2009) and Zdunik et al. (2004). In addition, similar calculations were done with a broad set of realistic EoS in Salgado et al. (1994).

Effects of rotation on the backbending phenomenon in NS were considered before in Zdunik et al. (2006), Chubarian et al. (2000), Cheng, Yuan \& Zhang (2002) and Heiselberg \& Hjorth-Jensen (1998). As the stars spin-down due to the loss of angular momentum, the central density increases and a phase transition to pure quark matter might occur (Glendenning et al. 1997; Cheng et al. 2002). In order to investigate if our EoS produces similar mass-radius diagrams as in Zdunik et al. (2006), in Fig. 2, we show the baryonic mass as a function of the circular equatorial radius for NS with frequencies ranging from 0 to $1200 \mathrm{~Hz}$. For similar mass-radius diagrams $M_{\mathrm{B}}\left(R_{\text {eq }}\right)$, see also Zdunik et al. (2004). In this case, it was

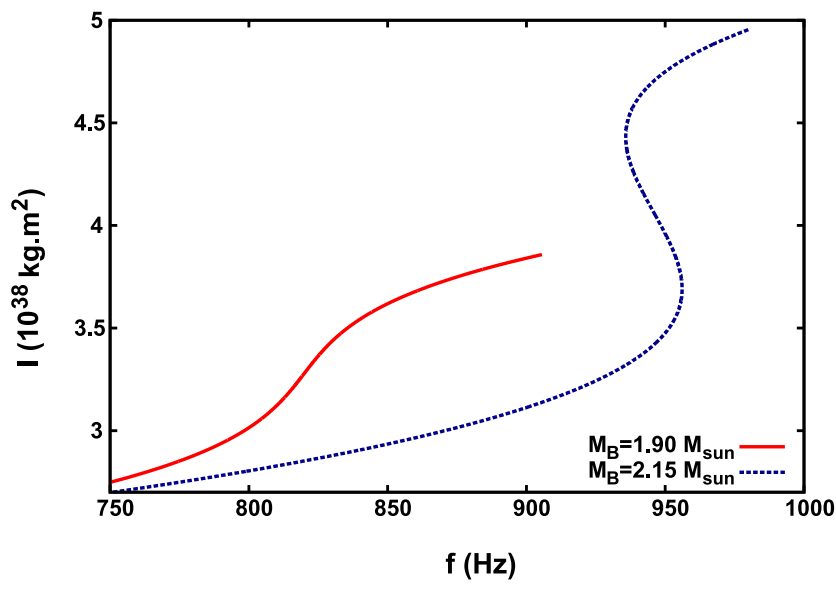

Figure 3. Relation between the moment of inertia and the frequency for rotating neutron stars at different fixed baryon masses, 1.90 and $2.15 \mathrm{M}_{\odot}$. respectively.

shown that EoS with hyperon degrees of freedom can also produce the backbending phenomenon. In our case, we have neglected additional exotic phases with hyperons in order to investigate exclusively the effects of a quark-hadron phase transition.

According to Fig. 2, the mass-radius curves present inflexion points (with a minimum in the baryon mass $M_{\mathrm{B}}$ ) for frequencies between $\sim 900$ and $\sim 1200 \mathrm{~Hz}$. This defines a region (at fixed baryon masses) where the backbending phenomenon may appear in the stars. Conclusions along this line were already studied in Zdunik et al. (2006). The diagram in Fig. 2 shows that, as the frequency increases, both the baryon mass and the radius of the stars increase, which is a direct effect of the centrifugal forces due to rotation. We choose to show in Fig. 2 the baryon mass instead the usual gravitational mass, since this is the fixed quantity during the evolution of isolated NS.

In Fig. 3, we present the moment of inertia as a function of the rotational frequency $f$ for two different stars at fixed baryon masses of $M_{\mathrm{B}}=1.90$ and $2.15 \mathrm{M}_{\odot}$. Both the moment of inertia $I$ and the angular velocity $\Omega$ are decreasing functions of time. According to the Fig. 3 , for the star with $M_{\mathrm{B}}=1.90 \mathrm{M}_{\odot}$, there is a reduction in the spin-down rate when this star undergoes a phase transition. This effect is more pronounced in the case of $M_{\mathrm{B}}=2.15 \mathrm{M}_{\odot}$, in which the quark-hadron phase transition induces a spin-up era in the star's evolution. During this time, the star will lose energy due to dipole radiation but, still, it will spin faster and grow in size. This same effect was already reported in Glendenning et al. (1997) and Weber, Glendenning \& Pei (1997).

The spin-down relation of a pulsar can be written as $\dot{\Omega}=$ $-C \Omega^{n+1}$, where $C$ is a term related to the structure of the star and $n$ is the braking index, which can be obtained directly from the frequency $\Omega$ of the pulsar and its time derivatives, $\dot{\Omega}$ and $\ddot{\Omega}$. The braking index can be expressed through the relation (Gao et al. 2016):

$n=\frac{\Omega \ddot{\Omega}}{\dot{\Omega}^{2}}$.

The energy loss due to the emission of radiation can be represented by the equation:

$\frac{\mathrm{d} E}{\mathrm{~d} t}=\frac{\mathrm{d}}{\mathrm{d} t}\left(\frac{1}{2} I \Omega^{2}\right)=-C \Omega^{n+1}$. 


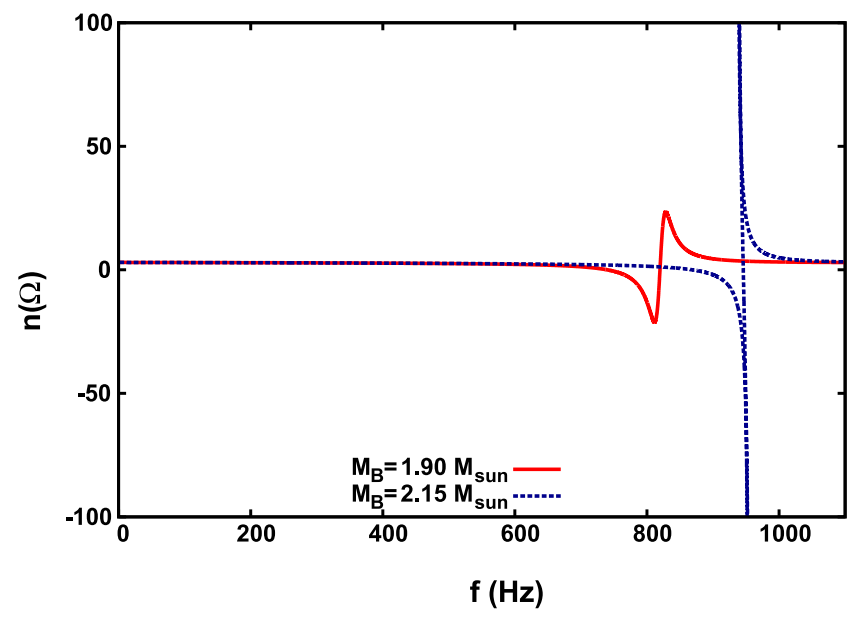

Figure 4. Braking index as a function of the frequency for rotating neutron stars. The curves correspond to the same stars as shown in Fig. 3

From equation (8), one can rewrite equation (7) in the form:

$n(\Omega)=3-\frac{3 I^{\prime} \Omega+I^{\prime \prime} \Omega^{2}}{2 I+I^{\prime} \Omega}$,

with $I^{\prime}$ and $I^{\prime \prime}$ being the first and the second derivatives of the angular momentum with respect to the angular velocity $\Omega, \frac{\mathrm{d} I}{\mathrm{~d} \Omega}$ and $\frac{\mathrm{d}^{2} I}{\mathrm{~d}^{2} \Omega}$, respectively. As a result, the braking index is now written in a frequency-dependent manner, $n(\Omega)$.

If one ignores the changes in the moment of inertia during the spin-down evolution, it can be seen from equation (9) that a purely dipole radiation yields a braking index of 3 . However, few measurements of braking index of isolated NS are available in the literature (see e.g. Gao et al. 2016; Hamil et al. 2015 and references therein), and in all cases, one has $n<3$. In order to determine accurately the braking index $n$, it is necessary to have high-precision measurements of the angular velocity $\Omega$ and its corresponding time derivatives $\dot{\Omega}$, which show how stars are slowing down. For this reason, braking index observations are much easier for young pulsars, not only because they spin very fast, but also because the braking is not affected by low timing noise or glitches. In addition, for older pulsars, the measurements of $\dot{\Omega}$ and $\ddot{\Omega}$ might require many years and yield very small values.

In order to evaluate the braking index in presence of a quarkhadron phase transition, we make use of the rotating configurations already shown in Fig. 3. The results are depicted in Fig. 4. In this case, the braking index does not deviate from 3 for slow rotation. However, as the frequency increases, it can reach values far from 3. In addition, when the phase transition is reached in the core of the star, there is an anomalous behaviour in the braking index curve $n(\Omega)$, whose extreme case is seen for the higher mass case $M_{\mathrm{B}}=2.15 \mathrm{M}_{\odot}$, where the braking index reaches values from $-\infty$ to $+\infty$.

Besides rotation, strong surface magnetic fields can be observed in some NSs, with values up to $10^{15} \mathrm{G}$. In this case, one expects to find even stronger magnetic fields inside these stars. For example, according to the virial theorem, compact stars can support internal magnetic fields of up to $10^{18-20} \mathrm{G}$ (see e.g. Fushiki, Gudmundsson \& Pethick 1989; Lai \& Shapiro 1991; Cardall, Prakash \& Lattimer 2001; Ferrer et al. 2010), turning such stars into a distinguished species than conventional radio pulsars. In contrast to rotationpowered pulsars, the strongly magnetized NS rotate slowly and, therefore, are powered by their magnetic field, see for example Olausen \& Kaspi (2014).

\section{MAGNETIZED HYBRID STARS}

In this section, we discuss the effects of strong magnetic fields on the global properties of NS, which are subjected to a sharp quark-hadron phase transition in their interior. Stationary and axisymmetric stellar models are constructed with the same numerical procedure and mathematical set-up as in Bocquet et al. (1995), Chatterjee et al. (2015) and Franzon et al. (2015), where the coupled Einstein-Maxwell equations were solved in a self-consistent way by means of a pseudo-spectral method.

It was shown in Chatterjee et al. (2015) and Franzon et al. (2015) that the leading contribution to the macroscopic properties of strongly magnetized stars, like mass and radius, originates from the pure field contribution to the energy-momentum tensor. In addition, the inclusion of magnetic fields effects in the EoS and the interaction between the magnetic field and matter (the magnetization) do not affect the stellar structure considerably. In this context, we do not take into consideration the magnetic field effects in our EoS.

According to Bocquet et al. (1995) and Cardall et al. (2001), the magnetic field is generated by the azimuthal component the electromagnetic current 4 -vector $j^{u}$ :

$j^{\phi}=\Omega j^{\mathrm{t}}+(e+p) f_{0}$,

with $j^{\mathrm{t}}$ being the time component of the electric current, $\Omega$ the stellar angular velocity, $e$ the energy density and $p$ the isotropic contribution to the pressure. The magnetic stellar models are obtained by assuming a constant current functions $f_{0}$. As shown in Bocquet et al. (1995), other choices for $f_{0}$ different from a constant value are possible, however, they do not alter the conclusions qualitatively. Nevertheless, a more comprehensive study of the field changes and the corresponding variation of current distributions would be very desirable. Such an analysis, however, requires much more insight into the microscopics of the currents in the different hadronic and quark phases and is beyond the scope of this initial discussion of possible observable effects of field decay in highly magnetized stars. As one can see from equation (10), for different values of $f_{0}$, the electric current changes and, therefore, the intensity of the magnetic field in the star changes.

We show in Fig. 5 the mass-radius diagram for stars at different fixed magnetic dipole moments $\mu$ and different current functions $f_{0}$. From Fig. 5, the masses and the radii increase by increasing $\mu$ and $f_{0}$. This is an effect of the Lorentz force which acts outwards and against gravity and, therefore the stars increase in size and can support more mass. A star with $M_{\mathrm{B}}=2.15 \mathrm{M}_{\odot}$ would be represented by a horizontal line in Fig. 5; in other words, it corresponds to a set of evolutionary sequences with smaller magnetic dipole moments. In this case, the magnetic moment loss in can be related to the change of the magnetic flux strength and distribution in the star due to ohmic dissipation (Goldreich \& Reisenegger 1992; Heyl \& Kulkarni 1998).

The existence of the backbending phenomenon in fast rotating NS are determined by the combination of three quantities: the baryon mass $M_{\mathrm{B}}$, the total angular momentum $J$ and the rotational frequency $f$. Minimum values of $M_{\mathrm{B}}$ at fixed $f$ with a monotonic behaviour of $M_{\mathrm{B}}$ versus $J$ leads to the backbending phenomenon, see e.g. Zdunik et al. (2006, 2004). In parallel to this, we conclude that the backbending in highly magnetized NS depends also on three quantities: the baryon mass $M_{\mathrm{B}}$, the magnetic dipole moment $\mu$ 


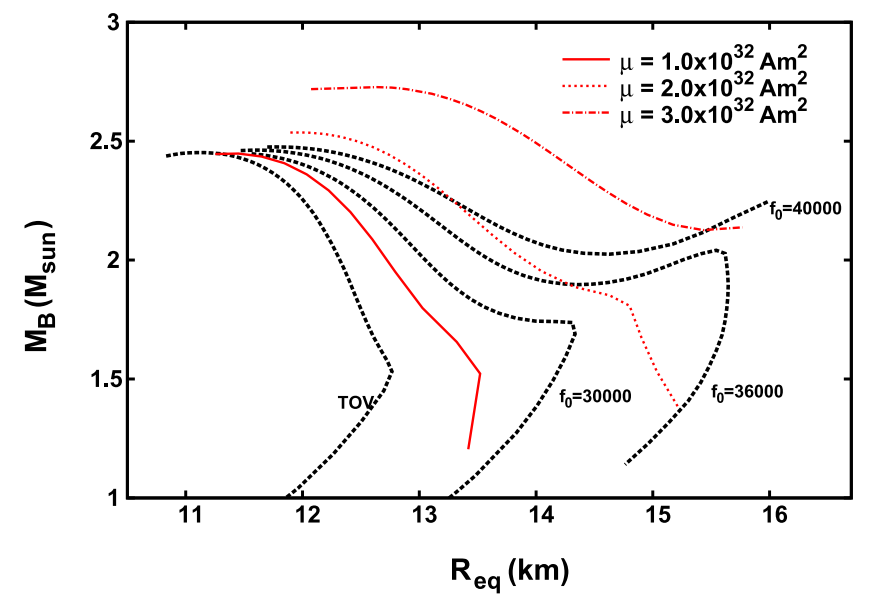

Figure 5. Mass-radius diagram for magnetized models. The calculations were done for different fixed current functions $f_{0}$ and different fixed magnetic dipole moments $\mu$.

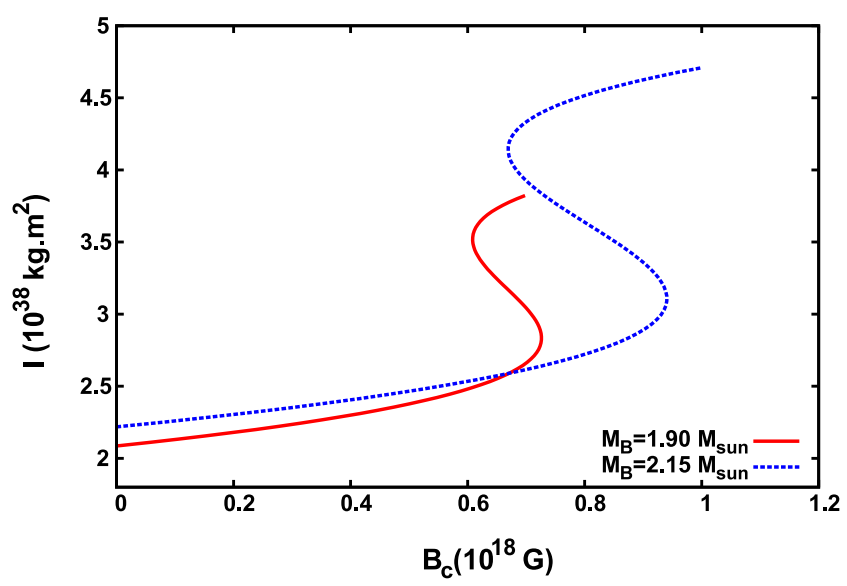

Figure 6. Relation between the moment of inertia and central magnetic field for magnetized neutron stars with different fixed baryon masses, 1.90 and $2.15 \mathrm{M}_{\odot}$, respectively.

and the current function $f_{0}$. In this case, the magnetic dipole loss leads to a quark-hadron phase transition inside the stars, followed by an increase in the electric current (and, therefore, the magnetic field) through the equation (10), which it is related to the change of the type of matter in the star with a different EoS. In contrast, rotating stars at fixed baryon masses have their frequency increased by angular moment loss during the backbending epoch.

As we already discussed for the case of rotation (but without magnetic field) in Fig. 3, the moment of inertia changes drastically from a constant value in the case of a more realistic treatment. In addition, a slower reduction of the moment of inertia, which is followed by a spin-up of the star, is observed when the EoS that describes the matter inside these objects includes a strong quarkhadron phase transition. We study the effect of magnetic fields on the moment of inertia $I$ for highly magnetized stars in Figs 6 and 7. We present $I$ as a function of the central and surface star magnetic fields, $B_{\mathrm{c}}$ and $B_{\mathrm{s}}$, respectively. These calculations are done for stars with the same fixed baryon masses as the ones presented in Fig. 3.

From Fig. 6, the higher the central magnetic field, the higher the moment of inertia of the stars. This effect is due to the Lorentz force which allow stars to support more mass. In addition, the circular equatorial radius of the sequence increases, as can be seen

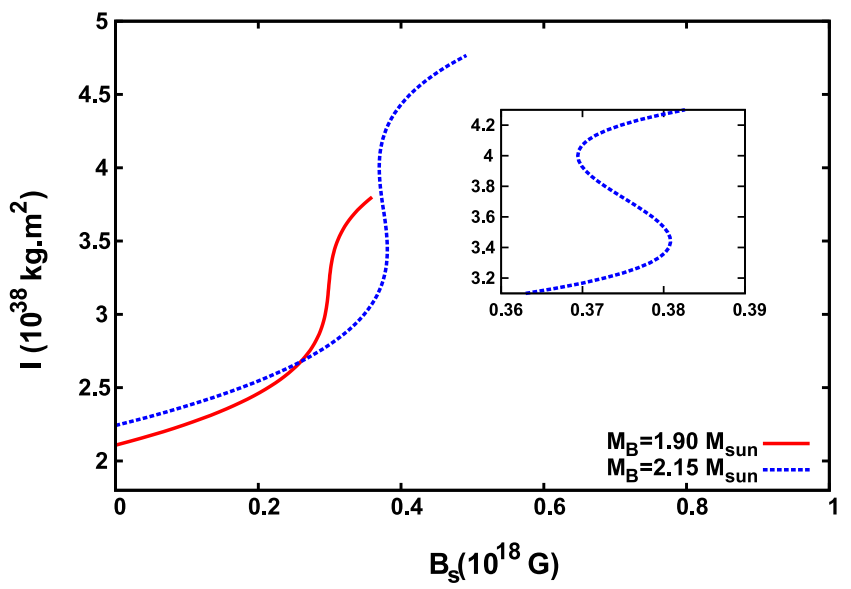

Figure 7. Same as in Fig. 6 but as a function of the surface magnetic field.

in the mass-radius diagram in Fig. 5 for higher current functions or magnetic dipole moments. According to Fig. 6, the maximum central magnetic field reached in stars depends strongly on the stellar mass. For example, a star with $M_{\mathrm{B}}=1.90 \mathrm{M}_{\odot}$ has a maximum central magnetic field of $\sim 7.0 \times 10^{17} \mathrm{G}$, whereas the star with $M_{\mathrm{B}}=2.15 \mathrm{M}_{\odot}$ can have a central magnetic field up to $1.0 \times 10^{18} \mathrm{G}$.

If knowing that the magnetic field decays over time, and fixing the baryon mass, each curve depicted in Fig. 6 represents the time evolution of the stellar magnetic field and moment of inertia of a different star. In other words, younger stars decrease in size and as the magnetic field decays, the central density increases, see e.g. Franzon et al. (2015). During this time, these stars might change from a hadronic to a quark phase in the core. In particular, the increase of the central magnetic field as shown in Fig. 6 may represent a signature of a phase transition inside these objects. However, internal magnetic fields cannot be directly constrained by observation. For this reason, we present in Fig. 7 the same star configurations as shown in Fig. 6, but as a function of the (polar) surface magnetic field, which can potentially be observed.

Looking at the star with fixed baryon mass of $1.90 \mathrm{M}_{\odot}$ in Fig. 7, one sees that the moment of inertia, which decreases in time, has a slower reduction when the stars passes through the phase transition. However, for the star with baryon mass of $2.15 \mathrm{M}_{\odot}$, the surface magnetic field, in fact, increases due to the phase transition. In this case, the moment of inertia as a function of $B_{\mathrm{S}}$ exhibits a small 'magnetic-up era', whereas this same effect is much more evident when the central magnetic field $B_{\mathrm{c}}$ is considered.

According to Fig. 6, during the 'magnetic-up era', an $M_{\mathrm{B}}=$ $2.15 \mathrm{M}_{\odot}$ star has increased the value of its central magnetic field by an amount of $0.23 \times 10^{18} \mathrm{G}$, while the surface magnetic field varies from $0.37 \times 10^{18}$ to $0.38 \times 10^{18} \mathrm{G}$ (see Fig. 7). For a star with $M_{\mathrm{B}}=$ $1.90 \mathrm{M}_{\odot}$, the central magnetic field increases by an amount of 0.1 $\times 10^{18} \mathrm{G}$, while the surface magnetic field always decreases. Such effects might be associated with giant flares presented in magnetars (Mallick \& Sahu 2014). In addition, properties of these objects, such as neutrino emission and, consequently, the stellar cooling, can be strongly affected by this variation in the magnetic field strength. Studies in this line are being performed.

By increasing the magnetic field strength, the stellar deformation is much more significant. As a result, the shape of the star will become more elongated and a topological change to a toroidal configuration can take place (Cardall et al. 2001). However, our current numerical tools do not handle toroidal configuration, setting a 


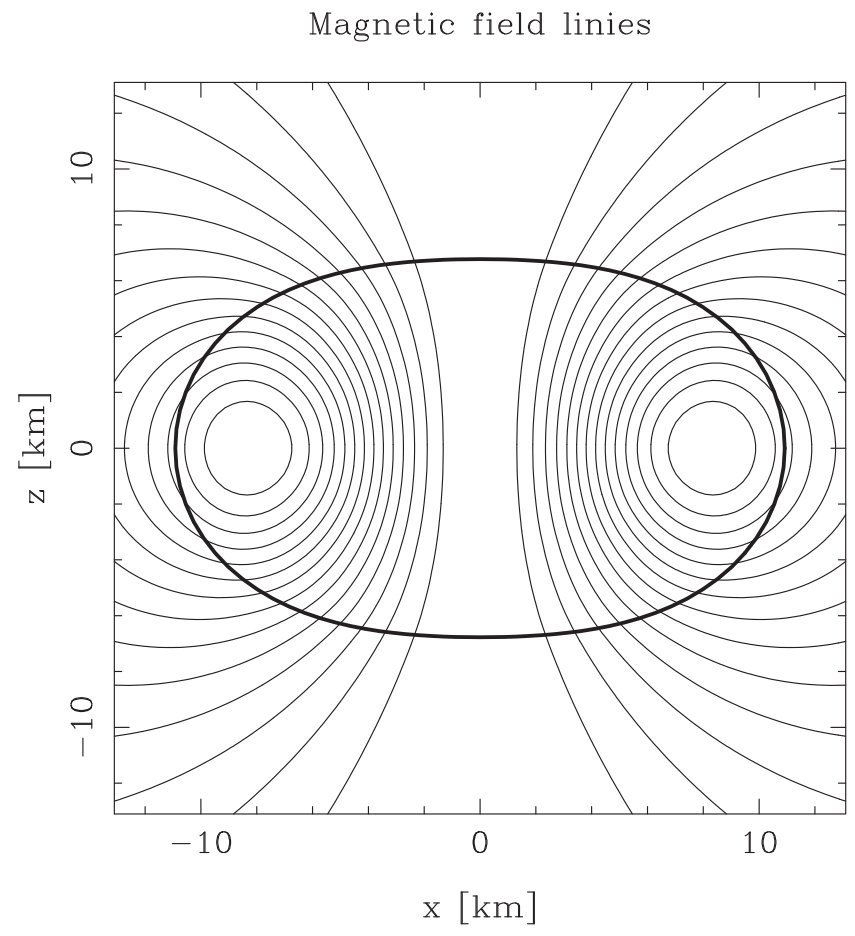

Figure 8. Magnetic field, i.e $A_{\phi}$, iso-contours measured by the Eulerian observer $\mathcal{O}_{0}$ for a star at fixed baryon mass of $2.15 \mathrm{M}_{\odot}$. This star is near the maximum equilibrium configuration achieved by the code with a magnetic dipole moment of $\mu=2.36 \times 10^{32} \mathrm{Am}^{2}$.

limit for the magnetic field strengths that we can obtain within this approach.

In order to see the deviation of spherical symmetry due to the anisotropy of the energy-momentum tensor in presence of strong magnetic fields, we show in Fig. 8 a star with $M_{\mathrm{B}}=2.15 \mathrm{M}_{\odot}$. The corresponding central baryon density is $n=0.45 \mathrm{fm}^{-3}$, with a gravitational mass of $1.92 \mathrm{M}_{\odot}$. The polar and the central magnetic fields are $4.62 \times 10^{17}$ and $1.03 \times 10^{18} \mathrm{G}$, respectively. The ratio between the magnetic pressure and the matter pressure at the centre of this star is 0.42 . This configuration is quite close to the maximum magnetic field configuration achieved with the code. One sees that the deviation from spherical symmetry is remarkable and it needs to be taken into account while modelling these highly magnetized neutron stars. Studies of relativistic models of magnetized stars with toroidal magnetic fields demonstrated also that the deviation from spherical symmetry is important, see Frieben \& Rezzolla (2012). Therefore, a simple Tolman-Oppenheimer-Volkoff general relativist solution (Oppenheimer \& Volkoff 1939; Tolman 1939) for these stars cannot be applied.

The deformation of magnetized NS can also be quantified by their quadrupole moment $Q$ with respect to the rotational axis. In Fig. 9, we show the quadrupole moment $Q$ as a function of the magnetic moment $\mu$ for a star at fixed baryon mass of $2.15 \mathrm{M}_{\odot}$. In Bonazzola \& Gourgoulhon (1996), it was found that in a low B-field approximation, $Q$ scales as $\mu^{2}$. From Fig. 9, one sees that indeed the quadrupole moment $Q$ grows parabolically for low polar magnetic fields. Still, according to Bonazzola \& Gourgoulhon (1996), the gravitational wave (GW) amplitude $h_{0}$ is given by

$h_{0}=\frac{6 G}{c^{4}} \frac{\Omega^{2}}{D} Q$,

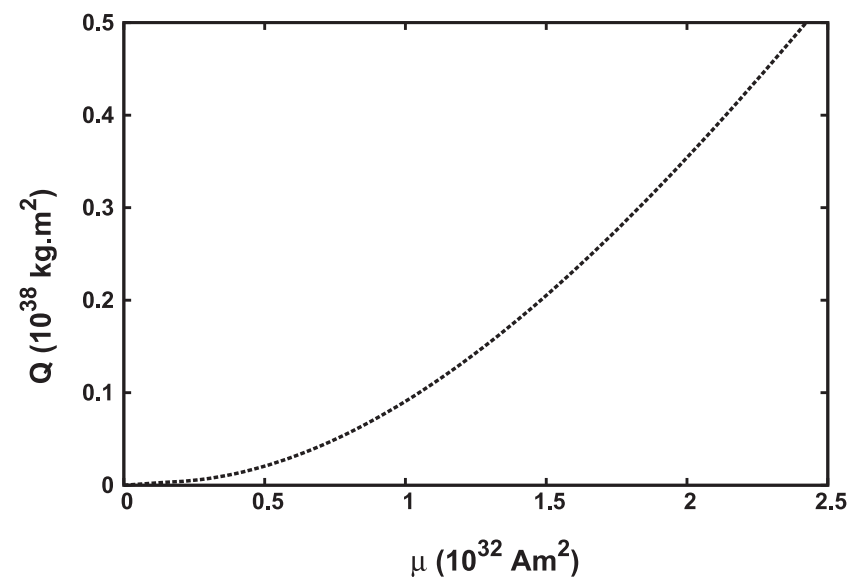

Figure 9. Quadrupole $Q$ as a function of the magnetic dipole moment for a star at fixed baryon mass of $2.15 \mathrm{M}_{\odot}$. This same star is depicted in Fig. 8 .

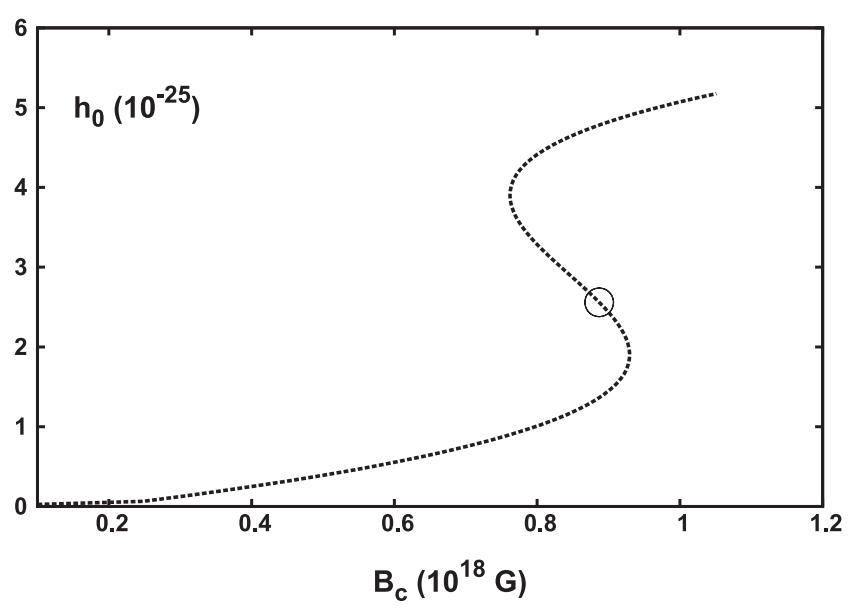

Figure 10. Gravitational wave amplitude as a function of the central magnetic field for the same configurations as in Figs 8 and 9.

with $G$ being the gravitational constant, $c$ the speed of light, $D$ the distance of the star and $\Omega$ the rotational velocity of the star.

In our models, the rotation and magnetic axes are aligned. In this case, even stars strongly deformed do not emit gravitational radiation. However, an estimate of the strength of GW emission can be deduced if we assume that the magnetic axis and the rotation axis are not aligned, as it seems to be the case, for example, in observable pulsars (Bonazzola \& Gourgoulhon 1996). This is a good approximation as long as rotational and magnetic field effects do not start to compete in deforming the star. For strong magnetic fields and as long as the rotation frequency is well below the Kepler frequency, this assumption should hold true.

We use our models to make a crude estimate of the GW strength in highly magnetized NS. Assuming that a star with $M_{\mathrm{B}}=2.15 \mathrm{M}_{\odot}$ rotates at a frequency of $f=\Omega / 2 \pi=1 \mathrm{~Hz}$ at a distance $D=$ $10 \mathrm{kpc}$, we obtain a GW amplitude of $h_{0}=2.56 \times 10^{-25}$ (see the black circle in Fig. 10), which could be measured by Laser Interferometer Gravitational-Wave Observatory (LIGO) and European Gravitational Observatory (EGO) interferometric detectors (Bonazzola \& Marck 1994; Bonazzola \& Gourgoulhon 1996).

In Fig. 10, we estimate the GW emission amplitude for different central magnetic fields. As already depicted in the relation between the moment of inertia $I$ and the magnetic field (see Fig. 6), we see that the GW amplitude $h_{0}$ can change significantly as the magnetic 
field decays over time showing a backbending behaviour as the star undergoes a quark-hadron phase transition. According to Fig. 10, the star may have a period of faster reduction in the GWs emission before the magnetic reduces completely. In Fig. 10, the black circle represents the same stars as depicted in Fig. 8.

\section{CONCLUSIONS}

In this work, we studied the effects of quark-hadron phase transition on the structure of NS, considering rotation as well as strong magnetic fields. The stellar models were obtained within a general relativity approach which solves the Einstein-Maxwell equations self-consistently by means of a spectral method. We employed an EoS that describes massive hybrid stars composed of nucleons, leptons and quarks $u, d$ and $s$. The hadronic phase was described in a relativistic mean field formalism, where many-body forces were taken into consideration. For the quark phase, we used the MIT bag model with a vector interaction. As in previous calculations (Chubarian et al. 2000; Zdunik et al. 2006), we showed that by employing this type of EoS with a sharp phase order transition, one obtains the backbending phenomenon.

The change from a confined to a deconfinement phase at the centre of NS leads to a drastic softening of the EoS. As a result, stars at fixed baryon masses decrease their gravitational masses (and the central pressure increases) during the evolutionary sequence. When the softening of the EoS is very pronounced, this leads to a sudden contraction of the neutron star at a critical angular velocity, which can be observed during the evolution of fast rotating and isolated pulsars through the spin-up era.

For stars at fixed baryon masses of 1.90 and $2.15 \mathrm{M}_{\odot}$, we have shown that, as they slow down due to the angular momentum loss through MDR, the internal density increases because of the weakening of the centrifugal force, leading the stars to reach the conditions for a phase transition in their interior. As a result of the phase change, the braking index changes dramatically around the frequency where the phase transition takes place, showing a divergent point.

We have also studied the effect of a quark-hadron phase transitions in NS with strong magnetic fields. We concluded that the mass-radius diagram and the shape of the star change significantly with the consistent inclusion of magnetic fields. The mass excess of the star is related to the Lorenz force, which increases with the magnetic field and therefore helps the star to support more mass than in the non-magnetized case. At the same time, the equatorial radii of theses stars increase, becoming much larger than their non-magnetized counterpart.

In parallel to the rotating case, we carried out relativistic calculations of the mass-radius diagram and the moment of inertia for hybrid stars endowed with strong magnetic fields. We have neglected the effect of the magnetic field in the EoS and of the magnetization of matter, since it was already shown that these contributions are very small even at large magnetic fields (Franzon et al. 2015). According to our results, in the same way that isolated pulsars have their frequencies increased in presence of a quark-hadron phase transition, the magnetic field can be amplified in highly magnetized hybrid NS. We performed calculations for stars at two different baryon masses, 1.90 and $2.15 \mathrm{M}_{\odot}$, respectively.

The relation $M_{\mathrm{B}}\left(R_{\mathrm{eq}}\right)$ presents similar behaviour in both rotating (and non-magnetized) and magnetized (and non-rotating) cases. For fast rotating pulsars, it is already known that the backbending phenomenon depends on three quantities: baryon mass $M_{\mathrm{B}}$, the angular momentum $J$ and the rotation frequency $f$. For strong magnetized stars, the increasing softening of the EOS leads to inflexions in the diagram $M_{\mathrm{B}}\left(R_{\mathrm{eq}}\right)$ versus $f_{0}$ with a monotonic behaviour at fixed magnetic dipole moments $\mu$. This is a similar condition expected to produce the backbending in isolated and fast rotating pulsars. In this case, the triple values $\left(M_{\mathrm{B}}, \mu, f_{0}\right)$ corresponds to $\left(M_{\mathrm{B}}, J, f\right)$.

The quark-hadron phase transition induces not only structural changes in NS, but can also modify their internal magnetic fields, what can affect, for instance, the thermal evolution processes. Magnetars have been observed to have very strong surface magnetic fields, $B_{\mathrm{s}}$. For this reason, we performed calculations of the moment of inertia as a function of $B_{\mathrm{s}}$. We have shown that the appearance of a 'magnetic-up era' is strongly related to the stellar mass. For these cases, we used a purely poloidal magnetic configuration with values for the surface magnetic fields beyond those observed. On the other hand, from virial theorem arguments, one can estimate a maximum limit for the magnetic field in the stellar interior and the values considered here agree with realistic situations.

We have also solved the Einstein-Maxwell equations and obtained the quadrupole moment $Q$ for slowly rotating magnetized NS. Although these stellar models have rotating and magnetic axes aligned and, therefore, do not emit gravitation waves, we use them to make a crude estimate of the gravitational radiation emitted by a star with $M_{\mathrm{B}}=2.15 \mathrm{M}_{\odot}$. The value obtained might lead to a detectable signal by VIRGO and LIGO. Note that the GW amplitude $h_{0}$ can be reduced rapidly in presence of a quark-hadron phase transition (see Fig. 10) inside stars with strong magnetic fields which, again, might be detected by VIRGO and LIGO.

As shown before in Markey \& Tayler (1973), Tayler (1973), Wright (1973) and Flowers \& Ruderman (1977), simple magnetic field configurations composed of purely poloidal or purely toroidal magnetic field configurations are always unstable. In addition, recent calculations showed that stable equilibrium configurations are possible only with magnetic fields configurations composed by a poloidal and a toroidal components (Braithwaite \& Spruit 2004; Braithwaite \& Nordlund 2006; Akgün et al. 2013; Armaza et al. 2015). Finally, the authors in Goldreich \& Reisenegger (1992) showed that ohmic decay, ambipolar diffusion, and Hall drift are responsible for the magnetic field decay in isolated NS. In this case, the magnetic field flux might be reduced and change its strength and distribution in the star (Srinivasan et al. 1990; Goldreich \& Reisenegger 1992; Heyl \& Kulkarni 1998). These points should be addressed in future investigations.

\section{ACKNOWLEDGEMENTS}

We gratefully acknowledge the referee for the valuable comments and suggestions, and Veronica Dexheimer for carefully reading the manuscript. B. Franzon acknowledges support from CNPq/Brazil, DAAD and HGS-HIRe for FAIR. S. Schramm acknowledges support from the HIC for FAIR LOEWE program. R.O. Gomes would like to thank the fruitful stay at FIAS. This work is partially supported by grant Nr. BEX 14116/13-8 of the PDSE CAPES and Science without Borders programs which are an initiative of the Brazilian Government. The authors wish to acknowledge the 'NewCompStar' COST Action MP1304.

\section{REFERENCES}

Akgün T., Reisenegger A., Mastrano A., Marchant P., 2013, MNRAS, 433, 2445

Alaverdyan A. G., Alaverdyan G. B., Chiladze A. O., 2010, Int. J. Mod. Phys. D, 19, 1557

Alford M., Braby M., Paris M. W., Reddy S., 2005, ApJ, 629, 969 
Alvarez-Castillo D. E., Blaschke D., 2015, Phys. Part. Nucl., 46, 846

Armaza C., Reisenegger A., Valdivia J. A., 2015, ApJ, 802, 121

Ayvazyan N. S., Colucci G., Rischke D. H., Sedrakian A., 2013, A\&A, 559, A118

Bhattacharyya A., Mishustin I. N., Greiner W., 2010, J. Phys. G, 37, 025201

Blandford R. D., Romani R. W., 1988, MNRAS, 234, 57P

Bocquet M., Bonazzola S., Gourgoulhon E., Novak J., 1995, A\&A, 301, 757

Bombaci I., Lugones G., Vidana I., 2007, A\&A, 462, 1017

Bombaci I., Logoteta D., Panda P. K., Providencia C., Vidana I., 2009, Phys. Lett. B, 680, 448

Bonazzola S., Gourgoulhon E., 1996, A\&A, 312, 675

Bonazzola S., Marck J., 1994, Annu. Rev. Nucl. Part. Sci., 44, 655

Bonazzola S., Gourgoulhon E., Salgado M., Marck J., 1993, A\&A, 278, 421

Braithwaite J., Nordlund $\AA .$, 2006, A\&A, 450, 1077

Braithwaite J., Spruit H. C., 2004, Nature, 431, 819

Brillante A., Mishustin I. N., 2014, Europhys. Lett., 105, 39001

Cardall C. Y., Prakash M., Lattimer J. M., 2001, ApJ, 554, 322

Chatterjee D., Elghozi T., Novak J., Oertel M., 2015, MNRAS, 447, 3785

Cheng K., Yuan Y., Zhang J., 2002, ApJ, 564, 909

Chubarian E., Grigorian H., Poghosyan G. S., Blaschke D., 2000, A\&A, 357,968

Contrera G. A., Spinella W., Orsaria M., Weber F., 2014, preprint (arXiv:1403.7415)

Cook G. B., Shapiro S. L., Teukolsky S. A., 1992, ApJ, 398, 203

de Carvalho S. M., Negreiros R., Orsaria M., Contrera G. A., Weber F., Spinella W., 2015, Phys. Rev. C, 92, 035810

Denke R. Z., Pinto M. B., 2013, Phys. Rev. D, 88, 056008

Dexheimer V., Negreiros R., Schramm S., 2015, Phys. Rev. C, 91, 055808

Duncan R. C., Thompson C., 1992, ApJ, 392, L9

Farhi E., Jaffe R. L., 1984, Phys. Rev. D, 30, 2379

Ferrari A., Ruffini R., 1969, ApJ, 158, L71

Ferrer E. J., de La Incera V., Keith J. P., Portillo I., Springsteen P. L., 2010, Phys. Rev. C, 82, 065802

Flowers E., Ruderman M. A., 1977, ApJ, 215, 302

Fraga E. S., Pisarski R. D., Schaffner-Bielich J., 2001, Phys. Rev. D, 63, 121702

Fraga E. S., Kurkela A., Vuorinen A., 2014, ApJ, 781, L25

Franzon B., Schramm S., 2015, Phys. Rev. D, 92, 083006

Franzon B., Dexheimer V., Schramm S., 2015, MNRAS, 456, 2937

Frieben J., Rezzolla L., 2012, MNRAS, 427, 3406

Friedman J. L., Ipser J. R., 1992, Phil. Trans. R. Soc. A, 340, 391

Fushiki I., Gudmundsson E., Pethick C., 1989, ApJ, 342, 958

Gao Z., Li X., Wang N., Yuan J., Peng Q., Du Y., 2016, MNRAS, 456, 55

Garcia A. F., Pinto M. B., 2013, Phys. Rev. C, 88, 025207

Glendenning N. K., 2012, Compact Stars: Nuclear Physics, Particle Physics and General Relativity. Springer-Verlag, New York

Glendenning N. K., Pei S., Weber F., 1997, Phys. Rev. Lett., 79, 1603

Gold T., 1975, Neutron Stars, Black Holes, and Binary X-ray Sources, Springer Verlag, 48, 354

Goldreich P., Reisenegger A., 1992, ApJ, 395, 250

Gomes R. O., Dexheimer V., Schramm S., Vasconcellos C. A. Z., 2015, ApJ, 808,8

Gourgoulhon E., 2012, 3 + 1 Formalism in General Relativity: Bases of Numerical Relativity, Vol. 846. Springer, Heidelberg

Haensel P., Zdunik J., Bejger M., Lattimer J., 2009, A\&A, 502, 605
Hamil O., Stone J., Urbanec M., Urbancov G., 2015, Phys. Rev. D, 91, 063007

Heiselberg H., Hjorth-Jensen M., 1998, Phys. Rev. Lett., 80, 5485

Hempel M., Pagliara G., Schaffner-Bielich J., 2009, Phys. Rev. D, 80, 125014

Heyl J. S., Kulkarni S., 1998, ApJ, 506, L61

Klähn T., Fischer T., 2015, ApJ, 810, 134

Komatsu H., Eriguchi Y., Hachisu I., 1989, MNRAS, 237, 355

Lai D., Shapiro S. L., 1991, ApJ, 383, 745

Lenzi C. H., Lugones G., 2012, ApJ, 759, 57

Lugones G., Grunfeld A. G., Al Ajmi M., 2013, Phys. Rev. C, 88, 045803

Mallick R., Sahu P. K., 2014, Nucl. Phys., A921, 96

Manchester R. N., Durdin J. M., Newton L. M., 1985, Nature, 313, 374

Markey P., Tayler R., 1973, MNRAS, 163, 77

Melatos A., 1999, ApJ, 519, L77

Menezes D. P., Pinto M. B., Castro L. B., Costa P., Providência C., 2014, Phys. Rev. C, 89, 055207

Olausen S., Kaspi V., 2014, ApJS, 212, 6

Oppenheimer J. R., Volkoff G. M., 1939, Phys. Rev., 55, 374

Ostriker J., Gunn J., 1969, ApJ, 157, 1395

Pacini F., 1967, Nature, 216, 567

Pacini F., 1968, Nature, 219, 145

Paczynski B., 1992, Acta Astron., 42, 145

Ranea-Sandoval I. F., Han S., Orsaria M. G., Contrera G. A., Weber F., Alford M. G., 2016, Phys. Rev. C, 93, 045812

Restrepo T. E., Macias J. C., Pinto M. B., Ferrari G. N., 2015, Phys. Rev. D, 91,065017

Salgado M., Bonazzola S., Gourgoulhon E., Haensel P., 1994, A\&A, 291, 155

Shao G. Y., Colonna M., Di Toro M., Liu B., Matera F., 2012, Phys. Rev. D, 85,114017

Shao G. Y., Colonna M., Di Toro M., Liu Y. X., Liu B., 2013, Phys. Rev. D, 87, 096012

Shapiro S. L., Teukolsky S. A., 2008, Black Holes, White Dwarfs and Neutron Stars: The Physics of Compact Objects. Wiley, New York

Spyrou N., Stergioulas N., 2002, A\&A, 395, 151

Srinivasan G., Bhattacharya D., Muslimov A., Tsygan A., 1990, Curr. Sci., 59,31

Stergioulas N., Friedman J. L., 1995, ApJ, 444, 306

Tayler R., 1973, MNRAS, 161, 365

Thompson C., Duncan R. C., 1993, ApJ, 408, 194

Thompson C., Duncan R. C., 1996, ApJ, 473, 322

Tolman R. C., 1939, Phys. Rev., 55, 364

Weber F., Glendenning N. K., Pei S., 1997, preprint (astro-ph/9705202)

Weissenborn S., Sagert I., Pagliara G., Hempel M., Schaffner-Bielich J., 2011, ApJ, 740, L14

Wright G., 1973, MNRAS, 162, 339

Yasutake N., Kashiwa K., 2009, Phys. Rev. D, 79, 043012

Yasutake N., Burgio G. F., Schulze H. J., 2011, Phys. Atom. Nucl., 74, 1502

Zdunik J. L., Haensel P., Gourgoulhon E., Bejger M., 2004, A\&A, 416, 1013

Zdunik J., Bejger M., Haensel P., Gourgoulhon E., 2006, A\&A, 450, 747

Zdunik J., Bejger M., Haensel P., Gourgoulhon E., 2008, A\&A, 479, 515

This paper has been typeset from a $\mathrm{T}_{\mathrm{E}} \mathrm{X} / \mathrm{L} \mathrm{T} \mathrm{E} \mathrm{X}$ file prepared by the author. 